\title{
A comprehensive synthetic genetic interaction network governing yeast histone acetylation and deacetylation
}

\author{
Yu-yi Lin, ${ }^{1,2}$ Yan Qi, ${ }^{1,3}$ Jin-ying Lu, ${ }^{1,4}$ Xuewen Pan, ${ }^{1,2,6}$ Daniel S. Yuan, ${ }^{1,2}$ Yingming Zhao, ${ }^{5}$ \\ Joel S. Bader, ${ }^{1,3}$ and Jef D. Boeke $e^{1,2,7}$ \\ ${ }^{1}$ High-Throughput Biology Center, Johns Hopkins University School of Medicine, Baltimore, Maryland 21205, USA; \\ ${ }^{2}$ Department of Molecular Biology and Genetics, Johns Hopkins University School of Medicine, Baltimore, Maryland 21205, \\ USA; ${ }^{3}$ Department of Biomedical Engineering, Johns Hopkins University, Baltimore, Maryland 21218, USA; ${ }^{4}$ Department \\ of Pharmacology and Molecular Sciences, Johns Hopkins University School of Medicine, Baltimore, Maryland 21205, USA; \\ ${ }^{5}$ Department of Biochemistry, University of Texas Southwestern Medical Center, Dallas, Texas 75390, USA
}

Histone acetylation and deacetylation are among the principal mechanisms by which chromatin is regulated during transcription, DNA silencing, and DNA repair. We analyzed patterns of genetic interactions uncovered during comprehensive genome-wide analyses in yeast to probe how histone acetyltransferase (HAT) and histone deacetylase (HDAC) protein complexes interact. The genetic interaction data unveil an underappreciated role of HDACs in maintaining cellular viability, and led us to show that deacetylation of the histone variant Htz1p at Lys 14 is mediated by Hda1p. Studies of the essential nucleosome acetyltransferase of $\mathrm{H4}(\mathrm{NuA4})$ revealed acetylation-dependent protein stabilization of Yng2p, a potential nonhistone substrate of NuA4 and Rpd3C, and led to a new functional organization model for this critical complex. We also found that DNA double-stranded breaks (DSBs) result in local recruitment of the NuA4 complex, followed by an elaborate NuA4 remodeling process concomitant with Rpd3p recruitment and histone deacetylation. These new characterizations of the HDA and NuA4 complexes demonstrate how systematic analyses of genetic interactions may help illuminate the mechanisms of intricate cellular processes.

[Keywords: Systems biology; histone; NuA4; acetylation; DNA repair]

Supplemental material is available at http://www.genesdev.org.

Received March 31, 2008; revised version accepted June 6, 2008.

Post-translational modifications of histones control many DNA-related processes (Kouzarides 2007). Dynamic histone (de)acetylation regulates gene transcription and silencing, chromosome condensation, DNA replication, and preservation of DNA integrity via DNA damage repair (Millar and Grunstein 2006). There are over 20 known histone acetyltransferases (HATs) and histone deacetylases (HDACs) in Saccharomyces cerevisiae; virtually all function as protein complexes (Lee and Workman 2007; Shahbazian and Grunstein 2007). These activities are coordinated in the cell, and comprise a system that dynamically regulates chromatin state. Systems with this many components are difficult to analyze using conventional genetics and biochemical methods, although some large-scale attempts have been made (Collins et al. 2007; Mitchell et al. 2008). Comprehensive assessment of this

${ }^{6}$ Present address: Verna and Marrs McLean Department of Biochemistry and Molecular Biology, Baylor College of Medicine, One Baylor Plaza, Houston, TX 77030, USA.

${ }^{7}$ Corresponding author.

E-MAIL jboeke@jhmi.edu; FAX (410) 502-1872.

Article is online at http://www.genesdev.org/cgi/doi/10.1101/gad.1679508. system is further complicated by the inclusion of essential genes (e.g., the essential acetyltransferase ESA1), requiring suitable conditional or hypomorphic query alleles. Moreover, recent studies in higher organisms have shown that HATs and HDACs have many substrates apart from histones (Glozak and Seto 2007; Xu et al. 2007), hinting that such substrates may exist in yeast as well.

Several recent studies have demonstrated that comprehensive genetic interaction profiling can effectively resolve complex pathways into conceptually and experimentally tractable modules (Tong et al. 2004; Schuldiner et al. 2005; Pan et al. 2006; Collins et al. 2007). Intergenic interactions can be either aggravating (negative), such as synthetic fitness or lethality defects (SFL), or alleviating (positive) such as synthetic rescue (SR). The genes involved can function either in a common essential pathway or in distinct but compensatory pathways converging on the same essential function (Hartman et al. 2001). Genetic interaction networks can be further organized into interacting functional modules based on a statistical analysis of the number of genetic interactions observed between sets of genes (Hartman et al. 2001; Tong et al. 
2004; Schuldiner et al. 2005; Segre et al. 2005; Pan et al. 2006; Collins et al. 2007).

Here we present a comprehensive genetic interaction network of HAT and HDAC protein complexes in yeast, generated using "diploid-based Synthetic Lethality Analysis on Microarray" (dSLAM) (Pan et al. 2006). The high degree of connectivity in this network enabled classification of interactions across protein complexes and identification of gene sets that function together as modules. Analysis of these modules revealed that histone hyperacetylation is as deleterious as hypoacetylation, consistent with previous studies showing that balanced acetylation status is crucial for cell viability (Vogelauer et al. 2000). Genetic interactions between HDAC complex (Carmen et al. 1996) and complexes involved in acetylation or deposition of the histone $\mathrm{H} 2 \mathrm{~A}$ variant $\mathrm{Htzlp}$ led us to demonstrate that HDA is the previously unidentified deacetylase for Htzlp. Other genetic interactions revealed that Esalp acetylates and stabilizes the nucleosome acetyltransferase of $\mathrm{H} 4$ (NuA4) subunit Yng2p, insights that helped formulate a new model for how the NuA4 complex undergoes rapid remodeling during the repair of DNA double-stranded breaks (DSBs). These new functions of the HDA and NuA4 complexes exemplify the value of comprehensive surveys of genetic interactions for exploring the roles of key protein complexes in controlling the dynamic balance of acetylation and deacetylation histones and other proteins.

\section{Results}

\section{Features of the interaction network}

We used dSLAM to determine genome-wide genetic interaction profiles of 38 query genes involved in histone (de)acetylation. Query genes included the HAT and HDAC catalytic subunits (Lee and Workman 2007) and associated protein complex subunits. Mutations used as queries included knockout deletions (KO) of 32 nonessential genes plus seven temperature-sensitive (Ts) or hypomorphic alleles of six essential genes (Fig. 1A; also see Supplemental Table S1 for a list of point mutations of the Ts and hypomorphic alleles). None of the essential genes had been used previously as query genes in genome wide studies. We validated 2823 unique pairwise genetic interactions involving 763 genes $(\sim 12.5 \%$ of yeast genes) by tetrad dissection and/or random spore analysis. There were comprise $105(\sim 4.2 \%)$ SR interactions, and 2718 SFL defects (Fig. 1A; see also Supplemental Fig. S1 for a high-resolution image of the entire clustogram; see Supplemental Table S2 for a complete list of genetic interaction pairs). Only $14 \%$ of the interactions identified here were reported previously. Essential query genes had 188 genetic interaction partners on average, whereas nonessential counterparts had 42, comparable with previous studies (Tong et al. 2004; Davierwala et al. 2005).

We arranged query genes by hierarchical clustering based on genetic interaction pattern similarities (Eisen et al. 1998). The essential query genes involved in histone acetylation formed a compact cluster, indicating that their patterns of genetic interaction were correlated (Fig.
1A). By contrast, essential genes involved in different biological processes shared little correlation with each other: Two examples are shown in Supplemental Figure S2 (CDC20 is the essential coactivator of anaphase-promoting complex, while $C D C 45$ is an essential DNA replication initiation factor). The interaction profiles identified using Ts alleles did not appear to be dominated by temperature effects, since the genetic interaction profile of esa1-Hm1, a hypomorphic allele of ESA1, with a lower growth rate than wild-type allele at $30^{\circ} \mathrm{C}$, most closely resembled that of the Ts allele esa1-531.

To discern relationships among genes at a higher level of organization, we examined interactions among sets of genes whose products might function together as a complex. We inferred membership in a complex from the subunit composition of known protein complexes or from highly interconnected patterns of genetic interactions indicating functional similarity. These complexes were regarded as nodes in a network in which the edges connected those pairs of nodes exhibiting more genetic interactions than expected by chance. Figure 2 shows a subnetwork highlighting HATs and HDACs (Supplemental Fig. S3 shows a comprehensive network; Supplemental Table S3 shows the complete data set). The resulting complex-to-complex network was highly connected. The close interactions among HATs and HDACs suggest that cells must maintain global acetylation levels within a certain range for viability. There were many aggravating interactions between different functional modules collectively comprising the same protein complex. Prominent SFL (blue) network hubs (see Supplemental Fig. S4 for degree distribution of network and hub definition) included the NuA4 and SAGA (Spt-Ada-Gen5-acetyltransferase) complexes and ACS2 (encoding an essential acetyl-CoA synthetase supplying the nucleocytosolic acetyl-CoA pool) (Takahashi et al. 2006).

The HDA complex was the most prominent alleviating (SR, red) hub, showing strong alleviating interactions with the NuA4, SAGA, and Elongator complexes. Not all deacetylase complexes had alleviating interactions with these complexes; for example, large and small forms of the $\operatorname{Rpd} 3$ complex $[\operatorname{Rpd} 3 \mathrm{C}(\mathrm{L})$ and $\operatorname{Rpd} 3 \mathrm{C}(\mathrm{S})$, respectively] had alleviating interactions only with $\mathrm{NuA} 4$ core acetylation machinery and aggravating interactions with most other HAT complexes, despite a broader histone substrate spectrum than the HDA complex. This finding suggests an unexpectedly detrimental effect of the HDA complex when the balance of chromatin acetylation and deacetylation is tilted. Moreover, the strong aggravating interaction between the HDA complex and Rpd3C mutants, and the fact that the growth defect and histone hyperacetylation of a hda1s rpd3s double mutant can be partially rescued by esa1-531 or gcn5s (Fig. $1 \mathrm{~B}, \mathrm{C})$, supports the idea that these two complexes are collectively (and redundantly) responsible for bulk cellular histone deacetylation, and suggests an overlooked role of deacetylation in maintaining cell viability.

Finally, our genetic interaction maps were enriched with genes annotated by Gene Ontology (GO) as having vacuolar/endosomal (Supplemental Fig. S5; see also Supplemen- 
Lin et al.

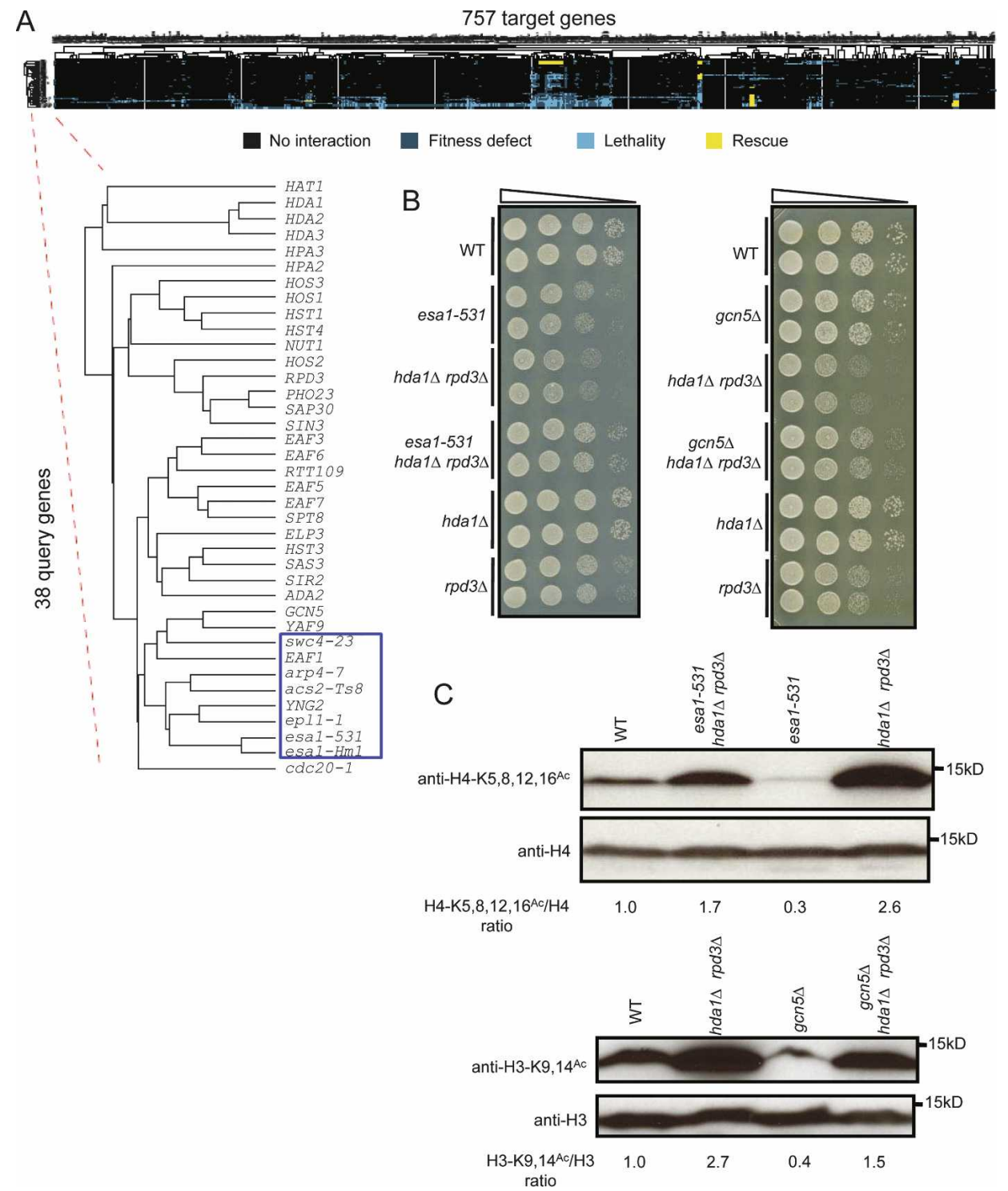

Figure 1. Global features of genetic interaction patterns. (A) Full hierarchical clustering of genetic interaction patterns. Blue, yellow, and black boxes represent aggravating, alleviating, and no interaction, respectively. Only validated data were used in hierarchical clustering and all subsequent computational analysis. The dendrogram of query genes was expanded for visualization, which indicated the similarities of their interaction patterns. The cluster of essential query genes is highlighted inside the blue box. $(B)$ esa1-531 and $g c n 5 \Delta$ partially rescue the growth defect of hda1s rpd3s double mutants. Growth of each strain was assessed by plating four 10-fold serial dilution on SC-Ura medium (for esa1-531 rescue experiment) or on YPD medium (for gcn5s rescue experiment) at $30^{\circ} \mathrm{C}$, a semipermissive temperature for esa1-531. (C) esa1-531 and gcn5s partially reversed hyperacetylation of histone $\mathrm{H} 4$ and $\mathrm{H} 3$, respectively, in hda1s rpd3s double mutant at $30^{\circ} \mathrm{C}$, a semipermissive temperature for esa1-531. The acetylation levels of $\mathrm{H} 4 \mathrm{~K} 5$, $\mathrm{H} 4 \mathrm{~K} 8$, $\mathrm{H} 4 \mathrm{~K} 12$, and H4 K16, and H3 K9 and H3 K14 were analyzed by immunoblot.

tal Table S4 for a full list of enriched GO annotations), which is also revealed in the comprehensive complexto-complex network (Supplemental Fig. S3). Similar enrichments have been observed in other studies (Takahashi et al. 2006; Mitchell et al. 2008). Previous expression microarray experiments of major HATs and HDACs (Choy and Kron 2002; Robyr et al. 2002; Le Masson et al. 2003; Huisinga and Pugh 2004; Zhang et al. 2004; Durant and Pugh 2006) identified no significant change of transcription of the key genes required for vacuolar/endo- somal function. The genetic interaction and transcriptional profiles suggest possible roles of these HATs and HDACs in regulating extranuclear functions through mechanisms other than regulation of transcription, recalling an apparent role of Elongator in regulating polarized exocytosis (Rahl et al. 2005), and the importance of the nuclear pore complex in controlling transcription (Akhtar and Gasser 2007) and the targeting of DNA DSBs to the nuclear periphery for efficient repair (N.J. Krogan, pers. comm.). 


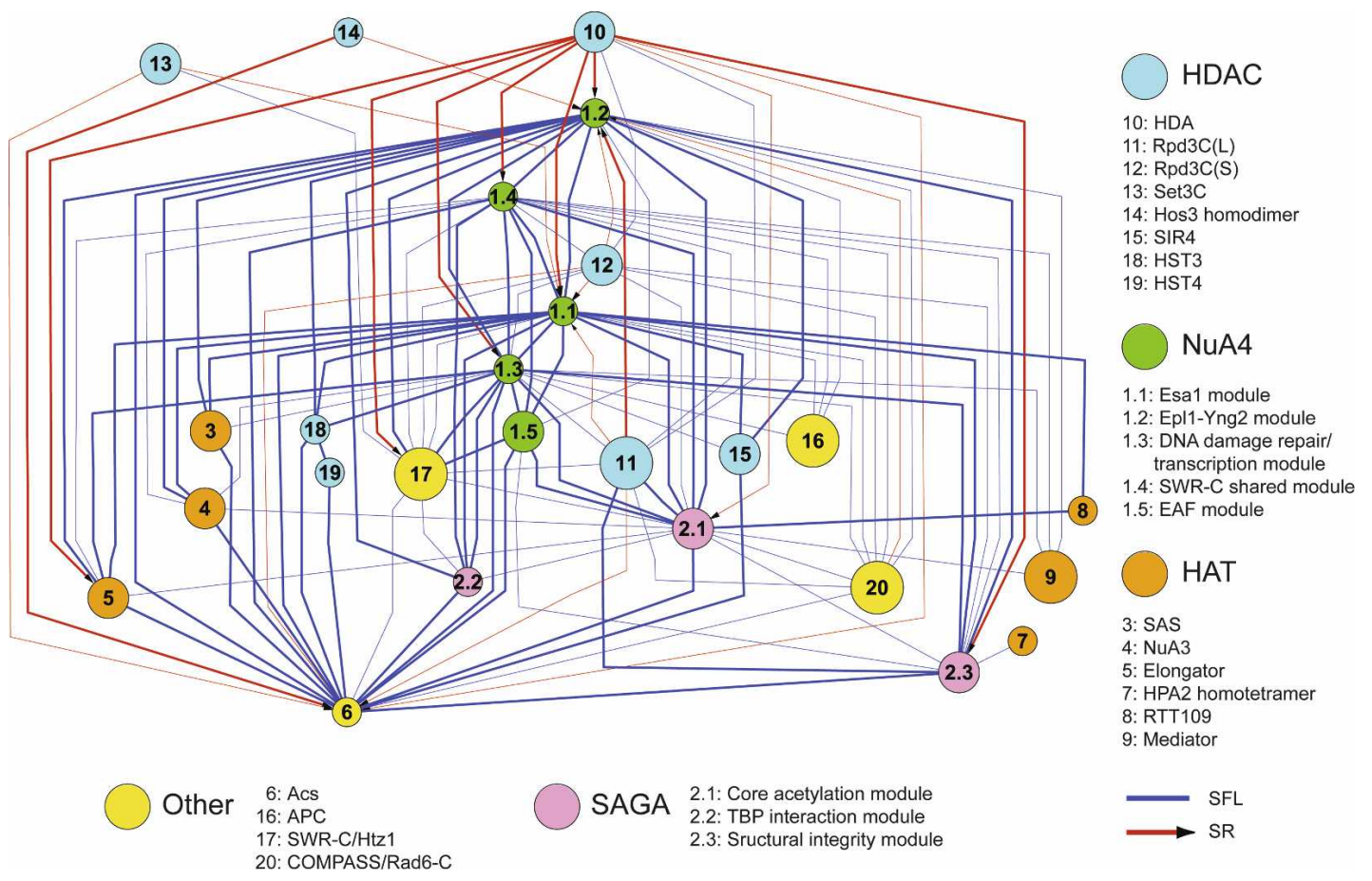

Figure 2. The derived complex-to-complex genetic interaction network for HATs and HDACs. Nodes represent protein complexes and functional modules. Pairs of nodes were linked by an edge if they had significantly more genetic interactions than expected by chance. Node sizes proportional to the number of subunits in the corresponding complex or module; edge thicknesses signifies the statistical significance of the enrichment in genetic interactions (thick and thin edges indicate $P<10^{-16}$ and $10^{-16} \leq P<10^{-5}$, respectively).

\section{HDA deacetylates Htz1-K14 ${ }^{A c}$}

Htzlp is an $\mathrm{H} 2 \mathrm{~A}$ variant isoform in yeast with important roles in transcription, DNA replication, chromosome segregation, and the delineation of heterochromatin boundaries (Santisteban et al. 2000; Meneghini et al. 2003; Krogan et al. 2004a; Dhillon et al. 2006). Htzlp is exchanged for H2A by the SWR-C chromatin remodeling complex (Krogan et al. 2003; Kobor et al. 2004; Mizuguchi et al. 2004), and acetylation of Htzlp is required for this process (Millar et al. 2006). Htzlp and SWR-C share similar genetic interaction patterns, and thus have been assigned to the same functional module (Krogan et al. 2003; Collins et al. 2007). It is known that NuA4 and SAGA acetylate Htzlp at all four lysine residues in its $\mathrm{N}$-terminal tail (K3, K8, K10, K14), with K14 being the most prominent acetylation site, but no Htzlp HDAC has been identified previously (Keogh et al. 2006; Millar et al. 2006). Htzlp-dependent genes tend to reside in small clusters called Htz1-activated domains (HZADs), and several of them overlap with Hda1-affected subtelomeric domains (HASTs) in subtelomeric chromatin (Meneghini et al. 2003). The two domains share the common feature of Hdalp-directed hypoacetylation of histone $\mathrm{H} 3$, which dampens the expression of their constituent genes.

Previous studies revealed synthetic lethality between $H T Z 1$ and three genes of NuA4 (EAF1, EAF5, and EAF7), as well as components of $\operatorname{Rpd} 3 \mathrm{C}(\mathrm{L})$ (Krogan et al. 2003;
Kobor et al. 2004). Our dSLAM data demonstrated strong aggravating genetic interactions between the SWR-C/ Htzlp module and all the functional modules of NuA4 and SAGA (Fig. 3A), as expected. We also observed a significant alleviating genetic interaction between the SWR-C/Htzlp module and the HDA complex (Fig. 3A). Deletion of $H D A 1$ rescued the slow growth phenotype of the $h t z 1 \Delta$ mutant and also reversed its sensitivity to the genotoxic agents hydroxyurea (HU) and methyl methanesulfonate (MMS) and to the microtubule-interfering drug benomyl (Fig. 3B). Transcription of many Htzlpdependent genes was also restored in the hda1s htz1s double mutant, including subtelomeric genes both in and out of HASTs and genes far away from telomeres (Supplemental Table S5). The ability of Htzlp to form a boundary adjacent to the silent mating-type cassette $H M R$ was preserved in the hda1s strain (Supplemental Fig. S6). In contrast to the other $h t z 1 \Delta$ phenotypes, deletion of HDA1 could not rescue the boundary function defect of $h t z 1 \Delta$ that limits spread of silent chromatin from $H M R$ locus (Supplemental Fig. S7; Meneghini et al. 2003).

Based on these observations, we investigated whether the HDA complex deacetylates Htzlp biochemically. Acetylation level of Htzlp at Lys 14 dramatically increased in vivo in the hda1s mutant, but not in any other HDAC deletion strain (Fig. 3C). Addition of affinity-purified Hdalp to Flag-tagged Htzlp purified from the $h d a 1 \Delta$ strain sharply diminished the increased level of K14 acetylation (Fig. 3D). Deletion of HDA1 also re- 
Lin et al.

Figure 3. HDA complex deacetylates Htz1-K14 ${ }^{\text {Ac }}$ in vitro and in vivo. (A) Overlap of the enzyme-substrate relationship with genetic interactions. A schematic model depicts genetic and enzyme-substrate interactions between HATs (NuA4 and SAGA), HDACs (HDA complex), and their substrates (Htzlp and histone $\mathrm{H} 3$ ). (B) Deletion of HDA1 rescues the slowgrowth and drug-sensitivity phenotype of $h t z 1 \Delta$. Four tetrads dissected from an

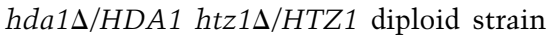
on YPD medium are shown. Drug sensitivities were assessed by plating 10 -fold serial dilution on YPD medium containing HU (200 mM), MMS (0.03\%), or benomyl $(15 \mu \mathrm{g} / \mathrm{mL}) . \quad(C, D)$ Hdalp deacetylates Htz1-K14Ac in vivo and in vitro. The K14 acetylation level in wild-type and HDAC mutant strains was analyzed by immunoblot. $(D)$ In vitro deacetylation activity of Hda1-TAP purified by tandem affinity purification was assessed by incubating it with Flag-tagged Htzlp purified from hda1s strain and then monitoring the K14 acetylation level by immunoblot. No protein was added to the control samples. $(E)$ Deletion of $H D A 1$ rescues the sensitivity of $h t z 1-K 14 R$ to benomyl. The experimental conditions are described in $B .(F) \mathrm{Ge}$ netic interactions of an $\mathrm{N}$-tail deletion of histone $\mathrm{H} 3[h h t 1(\Delta 1-36)]$ with hda1s (alleviating) and $h t z 1 \Delta$ (aggravating) mutants, suggesting that histone $\mathrm{H} 3$ reside in the same functional module as Htzlp.
A

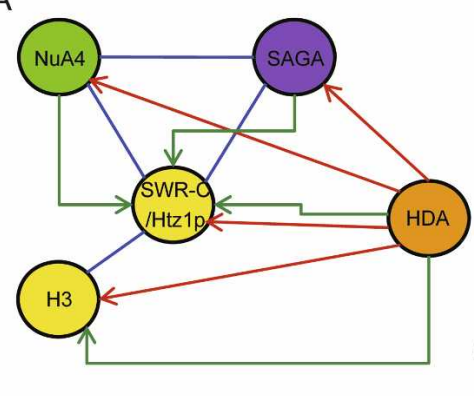

$\longrightarrow \mathrm{SFL} \rightarrow \mathrm{SR} \longrightarrow$ Enz-Sub

B
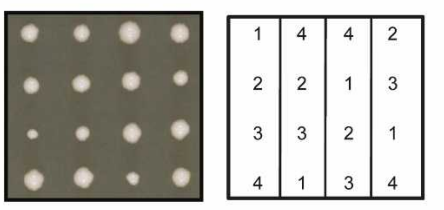

1 WT

2 hda1s

$3 h t z 1 \Delta$

$4 h d a 1 \Delta h t z 1 \Delta$
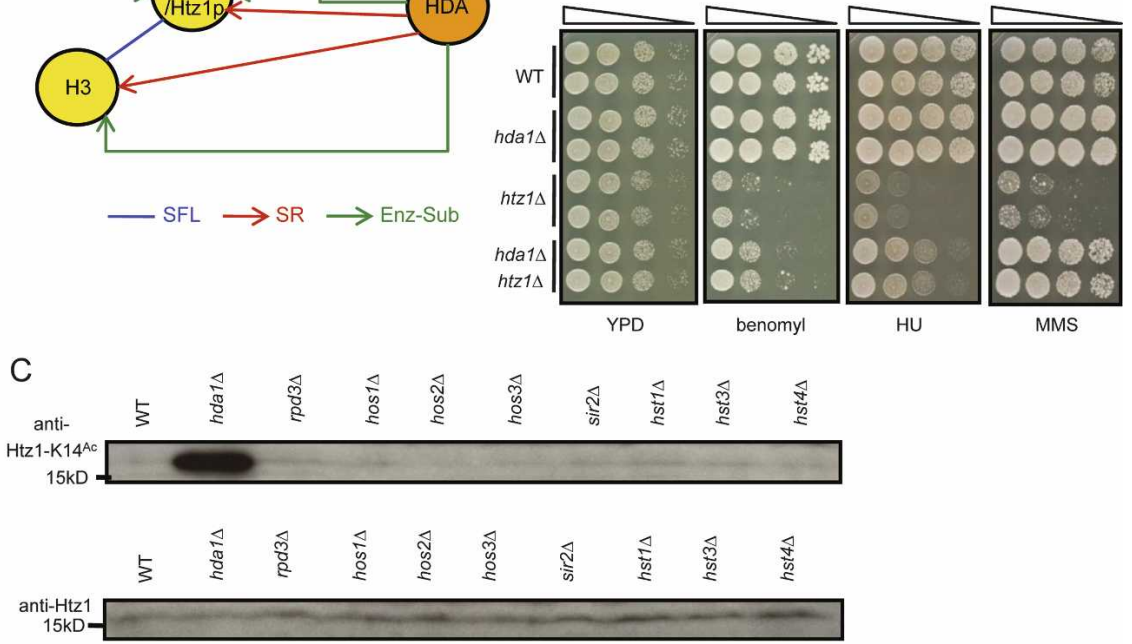

D

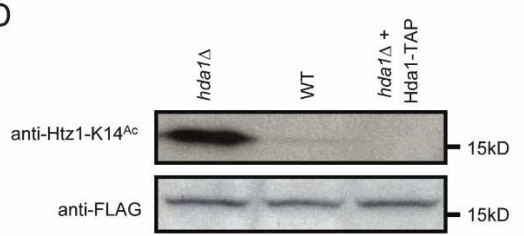

E
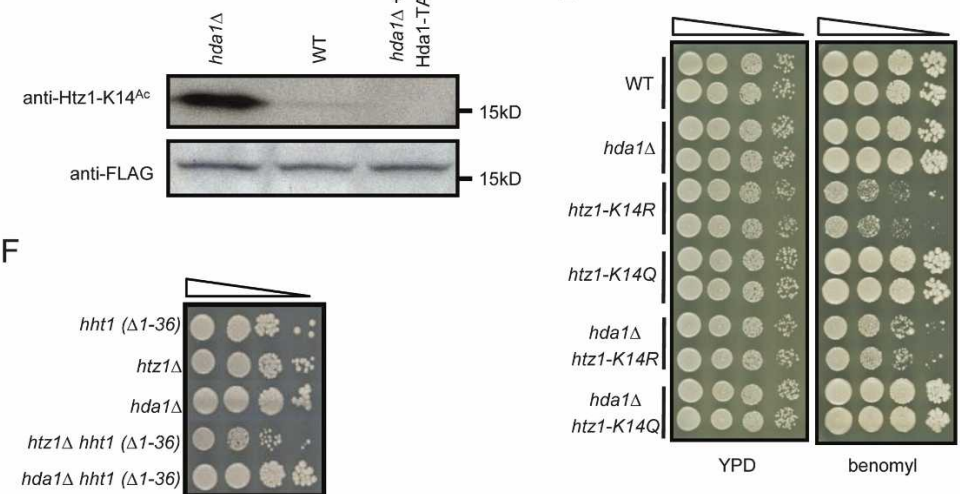

versed the sensitivity of $h t z 1-K 14 R$ to benomyl (Fig. 3E; Keogh et al. 2006), which suggests Hdalp may also deacetylate other lysine residues. These results showed that the HDA complex, previously regarded as an $\mathrm{H} 3$ and H2B-specific HDAC (Wu et al. 2001), also counteracts the effects of NuA4 and SAGA by removing a critical acetyl group from Htzlp.

The alleviating genetic interactions between hda1 and $h t z 1$ suggested the existence of parallel substrates for Hdalp, which would be predicted to display SFL interactions with the $h t z 1 \Delta$ mutant and SR interactions with the hda1s mutant. Histone H3 is a well-known substrate of HDA complex (Wu et al. 2001), and an N-tail deletion strain $[h h t 1(\Delta 1-36)]$ shows the expected genetic interaction pattern (Fig. 3F). Moreover, hht1 ( $\Delta 1-36)$ has genetic interaction with 88 out of 129 known genetic interaction partner genes of $h t z 1$ [see Supplemental Table S6 for a complete list of genetic interactions of $h h t 1$ ( $\Delta 1-36)$ and $h t z 1]$. These observations suggest that Htzlp, a histone $\mathrm{H} 2 \mathrm{~A}$ variant, and histone $\mathrm{H} 3$ share redundant functions.
Functional organization of NuA4-a new look

$\mathrm{NuA} 4$, the only essential HAT in yeast, acetylates the $\mathrm{N}$-terminal tails of $\mathrm{H} 2 \mathrm{~A}, \mathrm{H} 4$ and Htzlp. The processes are critical for regulating gene transcription, limiting the spread of silent heterochromatin and repairing DNA DSBs (Doyon and Cote 2004). Yeast cells lacking normal NuA4 arrest at the G2/M phase in a RAD9-dependent manner (Choy and Kron 2002). Esalp is the essential catalytic subunit of the NuA4 complex. It forms the core acetylation machinery with Epllp and Yng2p, termed Piccolo NuA4, to maintain global histone H4 and H2A acetylation in vivo (Boudreault et al. 2003; Selleck et al. 2005). Impaired function of any of the three component genes drastically decreases catalytic activity of the complex (Smith et al. 1998; Allard et al. 1999; Clarke et al. 1999; Choy and Kron 2002; Boudreault et al. 2003).

We found that the lethality of the esa1-531 and ep11-1 Ts alleles and the growth defect of yng2s could each be suppressed by deletion of either HDA1 or RPD3 (Fig. 2). 
In other words, growth defects resulting from impaired $\mathrm{NuA} 4$ acetylation were rescued by compensatory loss of either of the corresponding HDACs. We expected Ts mu- tants esa1-531 and epl1-1 to cluster most tightly but surprisingly, ep11-1 clustered with yng2s (Fig. 4A). Also, to our surprise, esa1-531 had a distinct response to the

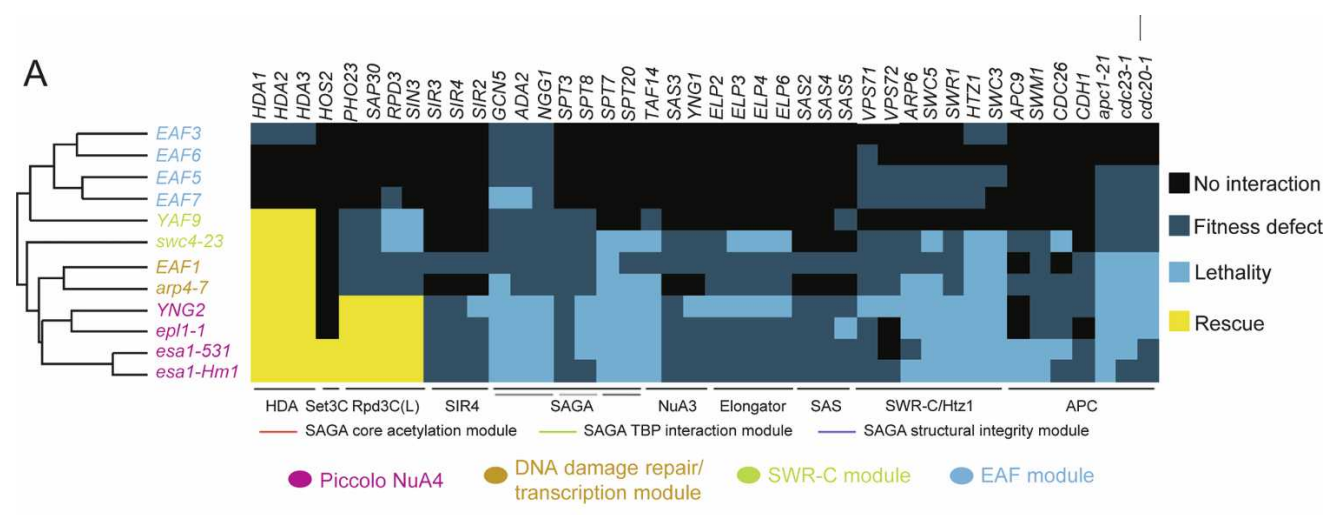

B

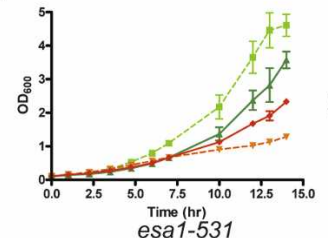

esa (hir) 531

C

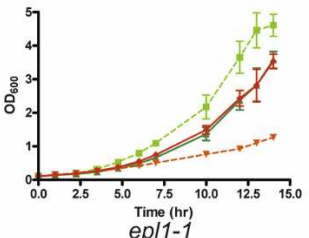

Time (hr)

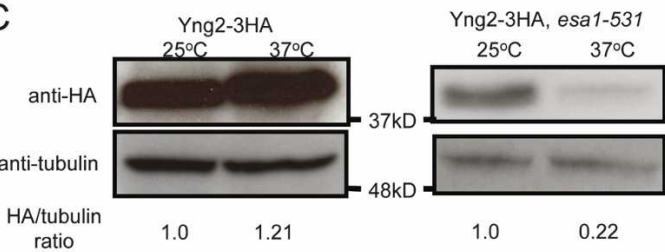

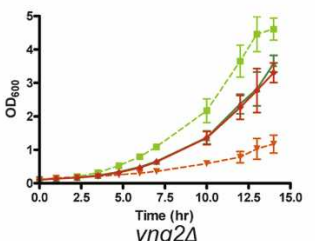

Time (hr)
yng2 $2 \Delta$

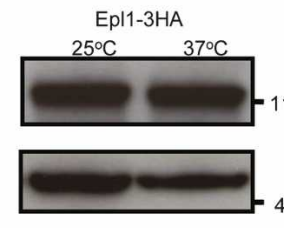

1.0

E

$$
\begin{aligned}
& \text {-를- WT } \\
& \leadsto \text { WT+TSA } \\
& -\overline{-}-\text { Mutant } \\
& \multimap \text { Mutant+TSA }
\end{aligned}
$$

Epl1-3HA, esa1-531 $25^{\circ} \mathrm{C} \quad 37^{\circ} \mathrm{C}$

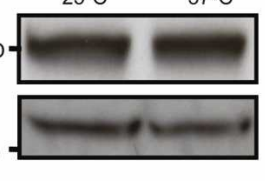

1.0

Yng2-3HA

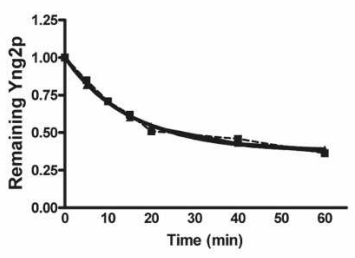

-WT $25^{\circ} \mathrm{C} \quad \-W T 37^{\circ} \mathrm{C}$
- - esa1-531 $25^{\circ} \mathrm{C} \rightarrow$ esa $1-53137^{\circ} \mathrm{C}$

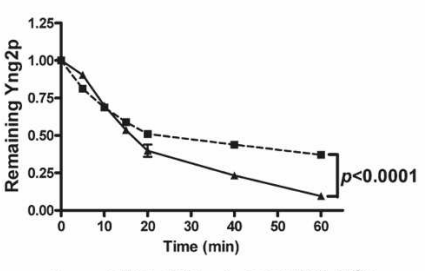
with MG-132

E

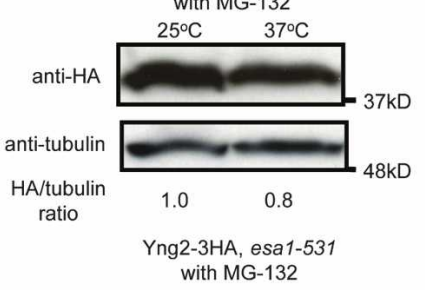

with MG-132 $25^{\circ} \mathrm{C} \quad 37^{\circ} \mathrm{C}$

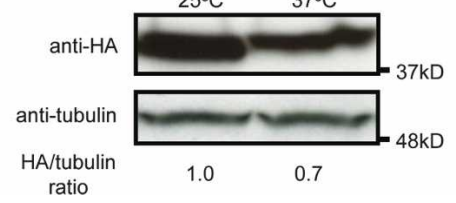

Figure 4. Functional dissection of the NuA4 complex. $(A)$ Hierarchical clustering of NuA4 component genes based on genome-wide genetic interaction patterns shows functional association. Font colors indicate distinct functional modules (see legend). Subsets of genetic interactions between NuA4 and other complexes were organized for visualization. $(B)$ Core acetylation machinery mutants are differentially sensitive to TSA. Growth curves of esa1-531, epl1-1, and yng2s mutants in SC-Ura medium at $37^{\circ} \mathrm{C}($ restrictive temperature) with or without TSA $(100 \mu \mathrm{M})$ are shown. Error bars indicate \pm 1 SEM from three biological replicates. (C) ESA1 stabilizes and controls the protein abundance of Yng2p. Wild-type (WT) and esa1-531 strains stably expressing tagged Yng2p or Epllp were grown at $25^{\circ} \mathrm{C}$ (permissive temperature) to $\mathrm{OD}_{600} \sim 0.3$, then shifted to $37^{\circ} \mathrm{C}$ or kept for $4 \mathrm{~h}$ at $25^{\circ} \mathrm{C}$; whole-cell extracts (WCEs) were then collected and probed with anti-HA, and band intensities were quantified. (D) Yng2-3HA turnover kinetics in wild-type and esa1-531 strains investigated by cycloheximide turnover experiments. Cells were grown at $25^{\circ} \mathrm{C}$ to $\mathrm{OD}_{600} \sim 0.3$, then either shifted to $37^{\circ} \mathrm{C}$ or kept at $25^{\circ} \mathrm{C}$, and treated with $0.1 \mu \mathrm{g} / \mathrm{mL}$ cycloheximide simultaneously. Equal amount of cells were collected at indicated time points and analyzed by immunoblot as shown in Supplemental Figure S9A. The fraction of Yng2-3HA remaining after cycloheximide addition was plotted. Error bars indicate \pm 1 SEM. (E) Yng2p is stabilized by MG-132 in esa1-531 cells. Wild-type and esa1-531 cells stably expressing tagged Yng2p were incubated with MG-132 $(75 \mu \mathrm{M})$ for $4 \mathrm{~h}$ at $25^{\circ} \mathrm{C}$ (permissive temperature) or $37^{\circ} \mathrm{C}$ (restrictive temperature), after which WCEs were analyzed by immunoblot. 
HDAC inhibitor trichostatin A (TSA) to that of epl1-1 and yng2 2 . TSA at $100 \mu \mathrm{M}$, which inhibits most HDAC activity of Hdalp and Rpd3p in vitro (Carmen et al. 1999), restored growth of ep11-1 and yng2s to that of wild type, whereas esa1-531 lethality was only partially rescued (Fig. 4B). This suggested a broader substrate spectrum for Esalp than for NuA4 per se; thus, Esalp might act independently of other NuA4 components. Conversely, nicotinamide (a chemical that inhibits another major type of HDAC named sirtuin) (Denu 2005) had little effect on the three mutants (Supplemental Fig. S8), and there were few SR interactions between sirtuin and NuA4 genes (Fig. 2).

Based on the distinct behavior of ESA1 relative to other genes encoding core NuA4 acetylation machinery, we hypothesized that Esalp performs functions beyond its well-established global chromatin acetylation activity in the context of Piccolo NuA4. We discovered that the abundance of Yng2-3HA (but not Epl1-3HA) depended on normal ESA1 function (Fig. 4C). We also showed that this effect involved the prevention of proteasome-mediated protein degradation (Fig. 4D; Supplemental Fig. S9A) and not transcriptional activation (Supplemental Fig. S9B), since Yng2 $p$ is stabilized by the proteasome inhibitor MG-132 (Fig. 4E).

To further investigate the mechanism of Esalp on regulating the protein stability of Yng2p, we examined the acetylation status of Yng2-Myc by immunoprecipitation with mouse monoclonal anti-acetylated lysine. To our surprise, a large proportion of Yng2p was acetylated in vivo (Fig. 5A). The signal of Yng2p acetylation could be efficiently competed away with acetylated BSA (Supplemental Fig. S10A), and mouse monoclonal antiHA could not pull down acetylated Yng2p to any detectable level (Supplemental Fig. S10B), indicating the specificity of the detected acetylation signals. The acetylation level of Yng2p diminished dramatically in an esa1-531 mutant at restrictive temperature (Fig. 5A), suggesting its acetylation depends on normal ESA1 function. A candidate lysine residue (K170) of Yng2p for acetylation was identified by tandem mass spectrometry (Supplemental Figure S11), which was confirmed by the loss of acetylation of Yng2p when substituting K170 with arginine (K170R), a mutation blocking acetylation (Fig. 5B). A K170R mutation constitutively destabilized Yng2p, whereas substituting K170 with glutamine (K170Q, a mutation mimicking constitutive acetylation) did not cause detectable change of protein abundance relative to the wild type, but rendered it insensitive to the effects of an esa1-531 Ts mutation (Fig. 5C). The K170R and K170Q mutants were each hypersensitive to benomyl and MMS (Fig. 5D), consistent with dynamic acetylation and deacetylation of Yng2p affecting its normal function. A screen of several known HDAC mutants identified in-
Figure 5. Acetylation and deacetylation of Yng2p controls its protein stability and function. (A) Yng2p is acetylated in vivo through an Esalp-dependent mechanism. Wild-type (WT) and esa1-531 strains stably expressing Myc-tagged Yng2p were grown at $25^{\circ} \mathrm{C}$ (permissive temperature) to $\mathrm{OD}_{600} \sim 0.3$, then shifted to $37^{\circ} \mathrm{C}$ or kept for $4 \mathrm{~h}$ at $25^{\circ} \mathrm{C}$; WCEs were then collected, immunoprecipitated with anti-Ac-K and probed with anti-Myc. (B) K170 is the major acetylated lysine residue of Yng2p in vivo. K170 was identified by tandem mass spectrometry. Substitution of K170 with arginine (K170R) diminishes acetylation of Yng2p. (C) Effects of K170 substitutions on Yng2p stability. Cells were grown in conditions describes in Figure 3A. WCEs were collected and probed with anti-HA, and band intensities were quantified. K170R mutation causes decreased protein abundance of Yng2p, while K170Q causes no detectable change. $(D)$ Effects of K170 substitutions on Yng2p function. Drug sensitivities were assessed by plating 10fold serial dilution on YPD medium containing MMS $(0.03 \%)$ or benomyl (15 $\mu \mathrm{g} /$ $\mathrm{mL})$. (E) Yng2p is deacetylated through an Rpd3p-dependent mechanism in vivo. Cells were grown at $30^{\circ} \mathrm{C}$ to $\mathrm{OD}_{600} \sim 0.6$, then WCEs were collected, immunoprecipitated with anti-Ac-K and probed with anti-Myc.

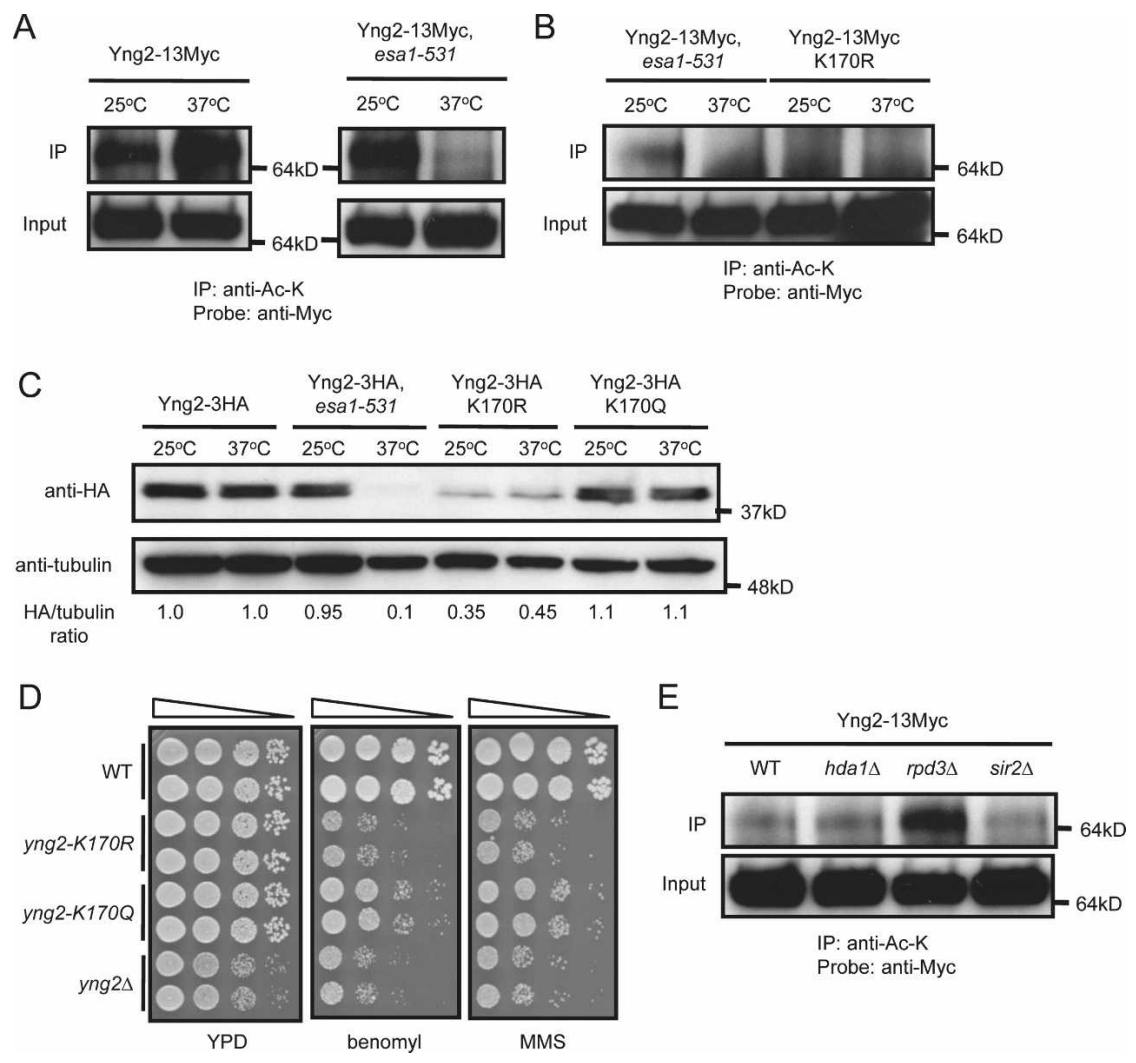


creased acetylation of Yng2p in $r p d 3 \Delta$ mutant (Fig. 5E), suggesting that deacetylation of Yng2p in vivo depends on the balance of normal ESA1 and RPD3 activities.

The genetic interaction profile of other NuA4 components provided additional information on the functional organization of the NuA4 complex (Fig. 4A). Arp4p is an actin-related gene shared among the NuA4, Ino80, and SWR-C chromatin remodeling complexes, and Arp4p is important for recruiting NuA4 to DSBs and specifically regulating the transcription of ESA1-dependent genes (Galarneau et al. 2000; Downs et al. 2004). ARP4 was previously assigned to the same module with three other genes (ACT1, SWC4, and YAF9) also shared between NuA4 and SWR-C (Doyon and Cote 2004; Auger et al. 2008). However, our genetic interaction data indicated that $A R P 4$ has a closer functional relationship to EAF1 than to SWC4 and YAF9 (Fig. 4A). This finding agrees with previous chemical genomics surveys implicating EAF1 in DNA damage repair (Parsons et al. 2004). Based on this, we assign ARP4 and EAF1 to the DNA damage repair and transcription regulation module, keeping SWC4 and YAF9 in the SWR-C module.

\section{Molecular choreography at DSBS}

Given that Esalp-dependent acetylation and Rpd3p-dependent deacetylation controls the protein stability of Yng2p, which is essential for the enzymatic activity of Piccolo NuA4 on acetylating nucleosomal histones (Boudreault et al. 2003; Selleck et al. 2005), we investigated the influence of these post-translational modifications on DSB chromatin dynamics. We used a galactose-inducible DSB induction system to monitor protein species and chromatin state at the DSB. The DSB, introduced by $\mathrm{HO}$ endonuclease at the mating type locus $(M A T)$, can only be repaired by nonhomologous end joining in this strain (Lee et al. 1998). In agreement with a previous study (Downs et al. 2004), we found transient hyperacetylation of histone $\mathrm{H} 4$ followed by a distinct hypoacetylation phase near the DSB (Fig. 6A). This change and others reported below were observed near the break and $2 \mathrm{~kb}$ distal to it but not $10 \mathrm{~kb}$ away. The recruitment of NuA4 to DNA DSBs is important for local acetylation of histone $\mathrm{H} 4$ (Bird et al. 2002; Downs et al. 2004; Tamburini and Tyler 2005), but the recruitment kinetics of Esalp, Epllp, and Yng2p were distinct from that of bulk NuA4 (Fig. 6B; Supplemental Fig. S12). Whereas Eaf1p, the only NuA4specific subunit protein not shared with any other protein complex (Auger et al. 2008), remained steadily enriched near the DSB, Esalp, Epllp, and Yng2p were initially recruited with kinetics similar to Eaflp but were evicted from the broken chromatin region at distinct rates thereafter. Addition of MG-132, a proteasome inhibitor that prevents Yng2p degradation (Fig. 4E), greatly delayed eviction of Yng2 $p$ from the DSB, suggesting an ubiquitin based displacement mechanism (Fig. 6B). Consistent with altered Yng2p recruitment kinetics, MG-132 also inhibited Ac-H4 depletion (Fig. 6A). In contrast, MG-132 did not affect histone H3 eviction kinetics (Supplemental Fig. S13). Rpn11p, a metalloprotease sub- unit of the 19S regulatory particle of the 26S proteasome lid (Verma et al. 2002), is concomitantly recruited to the DSB, suggesting that the proteasome is responsible for degrading Yng2p locally at the DSB (Fig. 6C).

A dynamic system with $\mathrm{H} 4$ acetylation followed by deacetylation might be crucial for efficient DSB repair is suggested by the hypersensitivity of GAL-HO strains to MG-132 upon DSB induction by galactose (Supplemental Fig. S14). Rpd3p was recruited to DSBs preceding deacetylation of histone $\mathrm{H} 4$ and eviction of Yng2p (Fig. $6 \mathrm{C}$ ), and addition of MG-132 did not change the kinetics of Rpd3p recruitment (Supplemental Fig. S15). By contrast, Hdalp and Hos2p were not enriched at the DSB (Supplemental Fig. S16). These data suggest that in addition to conducting global nucleosomal acetylation, Piccolo NuA4 is elaborately remodeled independently of the rest of NuA4 locally at DSBs and that this molecular choreography is governed by dynamic post-translational modification occurring specifically at DSBs (Fig. 7).

\section{Discussion}

In this study we systematically surveyed the functional associations among genes that dynamically regulate histone acetylation and deacetylation in yeast, generating a comprehensive network of genetic interactions. The inclusion of six essential query genes significantly enhanced our ability to identify functionally important target genes.

Our analysis focused on the abstracted network of functional modules and protein complexes rather than those of individual genes. The network was highly connected, and revealed a close functional relationship between HAT and HDAC complexes, indicating that these complexes share common essential cellular functions despite the fact that most of them modify distinct spectra of histone lysine residues. The HDA complex was the most distinct SR hub, pointing to major counterbalancing effects on the NuA4, SAGA, and Elongator HAT complex, which indicates that the HDA complex removed the largest amount of acetyl groups. By contrast, the Rpd3C had aggravating interactions with most of the HAT complexes, except Piccolo NuA4; these interactions are consistent with the cooperation between $\mathrm{Rpd} 3 \mathrm{C}(\mathrm{S})$ and various HATs in regulating transcriptional elongation (Carrozza et al. 2005; Keogh et al. 2005; Li et al. 2007), and also the role of Rpd3C in governing histone $\mathrm{H} 4$ deacetylation following an acetylation conducted by NuA4 in the vicinity of DNA DSBs.

Our results also revealed the general finding that the HDA and Rpd3 complexes together define the major HDAC activities that counteract the HAT activity of the NuA4 and SAGA complexes, and that these complexes provide the bulk control of the dynamic balance of global histone acetylation and deacetylation essential to cell viability. Requirement of histone acetylation by various HATs for maintaining cell viability have been well studied (Smith et al. 1998; Zhang et al. 1998; Allard et al. 1999; Clarke et al. 1999; Howe et al. 2001). Our data suggest that 
Lin et al.

A

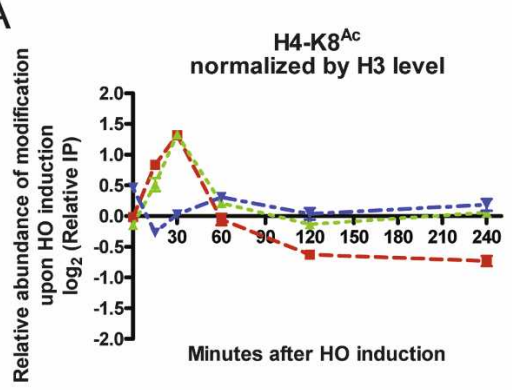

$\mathrm{B}$
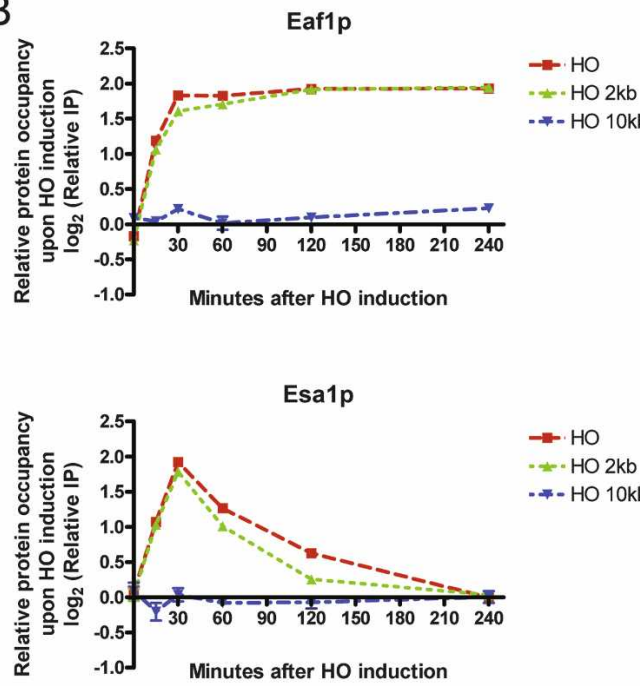

$\mathrm{C}$

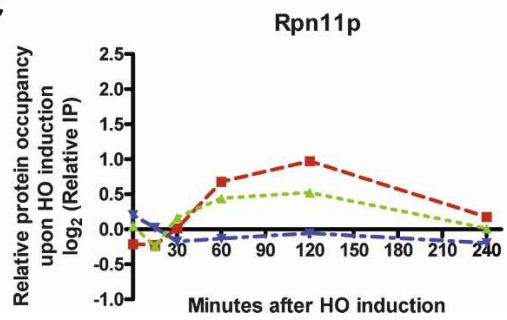

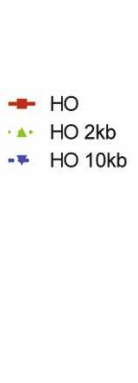
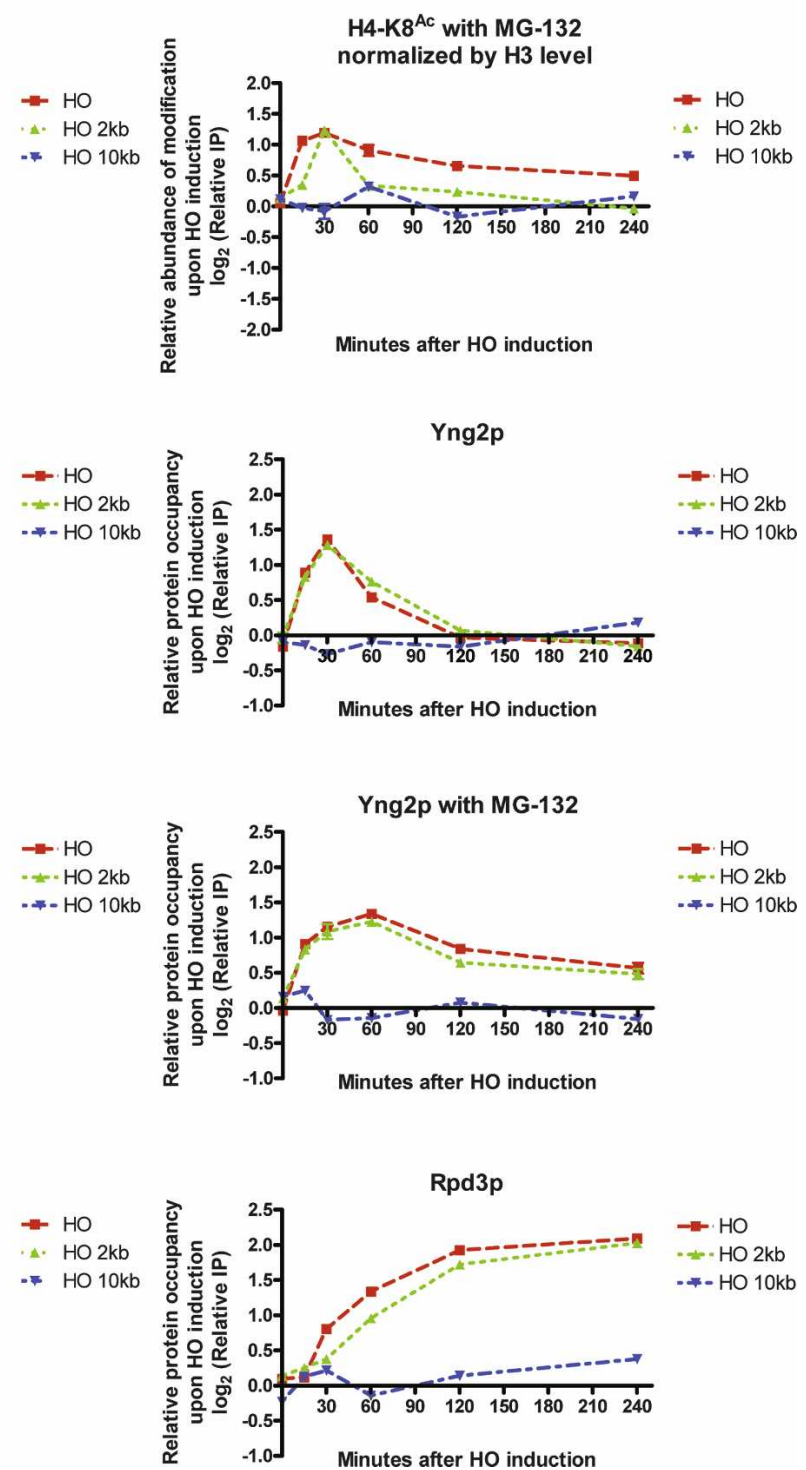

Figure 6. Post-translational protein modification of the NuA4 core acetylation machinery choreographs DSB repair. (A) MG-132 alters the kinetics of histone $\mathrm{H} 4$ acetylation near an HO-induced DNA DSB. Chromatin immunoprecipitation (ChIP) assays with anti-H4-K8 ${ }^{\mathrm{Ac}}$ were used to assess kinetics of histone H4 acetylation. Enrichment of histone H4-K8 ${ }^{\text {Ac }}$ was quantified by RT-PCR and normalized to the GEA2 internal control and also the local abundance of histone H3 (Supplemental Fig. S11). (B) NuA4 core acetylation machinery components show distinctive kinetics of recruitment to an HO-induced DNA DSB. The enrichment of tagged Esalp, Yng2p, and Eaflp after $H O$ induction was assessed by ChIP assays with anti-HA. GAL-HO pdr5 strains were used in MG-132 experiments. $(C)$ Proteasome and Rpd3C are recruited to an HO-induced DSB. Enrichment of tagged Rpn $11 \mathrm{p}$ and Rpd3p after $H O$ induction was assessed by ChIP with anti-HA.

hyperacetylation of histone $\mathrm{H} 3$ and $\mathrm{H} 4$ in the hda1s rpd3s double mutant is as detrimental to cell viability as hypoacetylation, and can be rescued when the responsible acetylase activity is repressed.

In addition to its effect on many aspects of chromosome biology, a relationship between global histone acetylation/deacetylation and vacuolar function is also revealed by these studies. This relationship raises the possible existence of nonhistone substrates of HATs and HDACs in yeast.

The genetic interaction profile of $h t z 1 \Delta$ and follow-up experiments led us to conclude that the HDA complex, previously known to acetylate histones $\mathrm{H} 3$ and $\mathrm{H} 2 \mathrm{~B}$, removes the acetyl group from K14 and possibly also other lysine residues of the N-tail of Htzlp. However, lack of reliable antibodies limited our ability to test the acetylation level of lysine residues other than K14. We also propose that histone $\mathrm{H} 3$ and Htzlp reside in the same functional module based on their similar genetic interaction profiles. However, although being less enriched in the promoter region and defective in blocking telomeric heterochromatin spreading, an unacetylatable Htzlp 


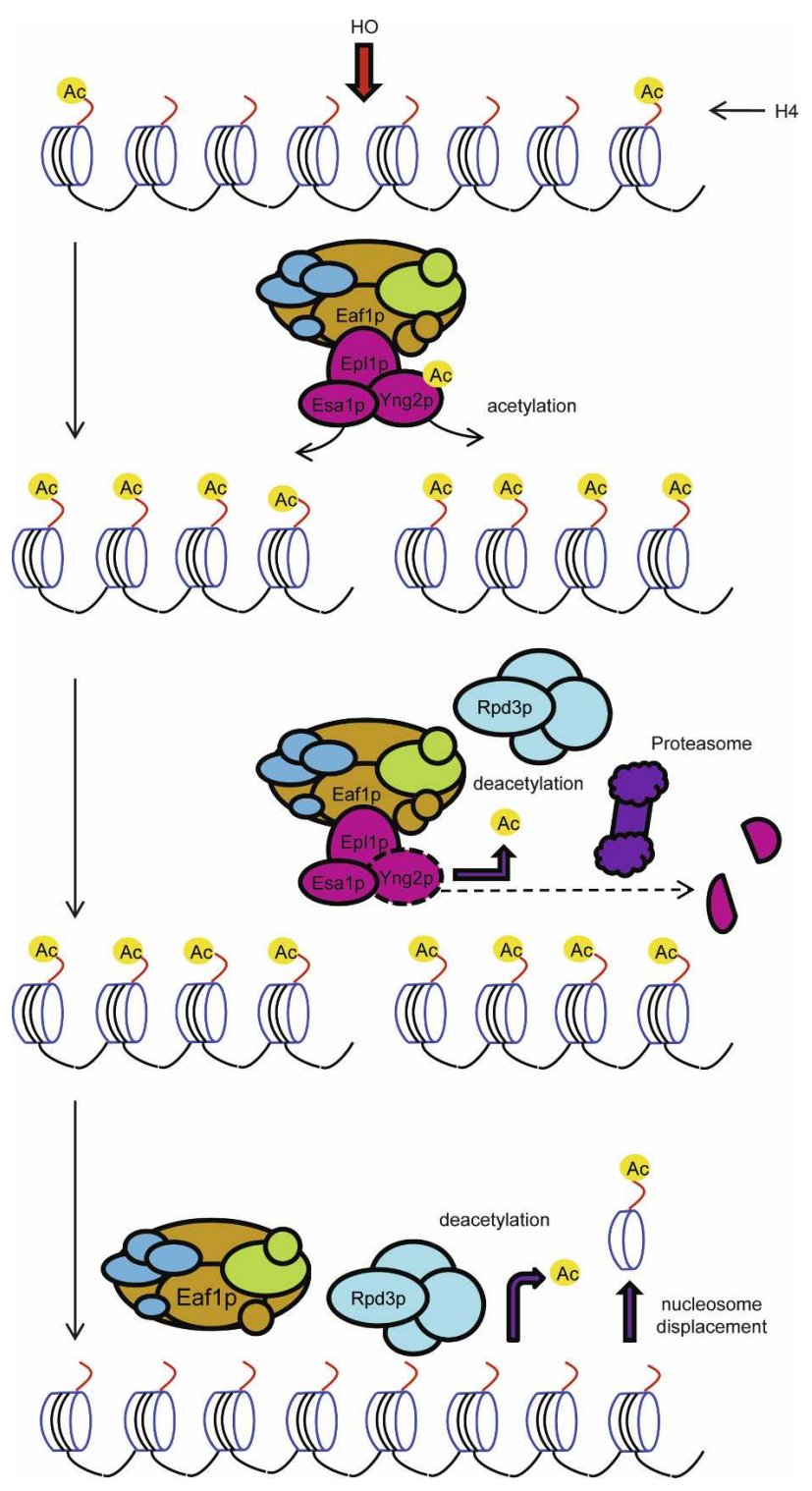

Figure 7. Schematic model for the dynamic regulation of acetylation status near DSBs. Upon introduction of a DSB, such as that caused by $\mathrm{HO}$ endonuclease at the $\mathrm{HO}$ recognition site, NuA4 is actively recruited focally and rapidly hyperacetylates nearby histone $\mathrm{H} 4$. The function of the core acetylation machinery of NuA4 is disrupted when Yng2p is deacetylated through an Rpd3C-depedent mechanism followed by degradation by proteasome, followed by eviction of Esalp and Epllp. Three distinct processes appear to be at play to facilitate transition to the hypoacetylation phase crucial for proper DSB repair: (1) The breakdown of Piccolo NuA4 stops ongoing acetylation, (2) Rpd3C is recruited and could actively remove acetyl groups, and (3) ATP-dependent chromatin remodeling complexes, which conduct active nucleosome displacement.

mutant $(h t z 1-K 3,8,10,14 R)$ is insensitive to genotoxic agents lethal to $h t z 1 \Delta$, suggesting that acetylation is important in some but not all aspects of Htzlp function (Babiarz et al. 2006; Millar et al. 2006). The genetic interactions between HTZ1 and its corresponding HATs (ESA1 and GCN5) and HDAC (HDA1) suggest that these complexes not only affect Htzlp modification but also modulate the essential pathway through additional unknown mechanisms.

In addition to examining functional relationships among protein complexes, synthetic genetic interaction profiles can be used for dissecting more elaborate protein complexes like NuA4 into separate functional modules. A recent genetic interaction survey of a subset of nonessential NuA4 subunits revealed that Eaflp is important for maintaining the integrity of NuA4 complex (Mitchell et al. 2008). Here, comprehensive incorporation of most essential and nonessential NuA4 subunit genes led to many new findings. The genetic interaction profile of esa1-531 revealed new functions of Esalp beyond its well-known nucleosomal acetylation activity. For example, we found that the enzymatic activity of Piccolo NuA4, the core acetylation machinery of NuA4, was maintained by control of Esalp on the protein turnover of Yng2p through an acetylation-dependent mechanism. Deacetylation of Yng2p was dependent on Rpd3p, which potentially precedes the degradation of Yng2p by proteasome. Tandem mass spectrometry and further biochemical experiments confirmed that K170 is the major acetylated lysine residue of Yng2p. The hypersensitivity of both yng2-K170R and yng2-K170Q mutants (mutations mimicking constitutive deacetylation and acetylation, respectively) to benomyl and MMS suggests that dynamic acetylation and deacetylation is important to its normal function in DSB repair. Moreover, the recruitment kinetics of the Piccolo NuA4 subunits to an HO-induced DSB is distinct from the rest of NuA4. NuA4 is actively recruited focally and rapidly hyperacetylates nearby histone $\mathrm{H} 4$ at a DSB. We propose that the dynamic protein turnover of Yng2p mediated by an acetylation-deacetylation cycle with cooperative recruitment of $\mathrm{Rpd} 3 \mathrm{C}$ and proteasome at DSBs disrupts the enzymatic activity of Piccolo NuA4, which stops ongoing acetylation. This finding is consistent with previous findings showing that proteasome is involved in the repair of DSBs (Krogan et al. 2004b). The recruited Rpd3C further removes acetyl groups from histone $\mathrm{H} 4$, and ATP-dependent chromatin remodeling complexes actively conduct nucleosome displacement (Tsukuda et al. 2005). These three mechanisms allow for dynamic hyperacetylation and subsequent hypoacetylation of histone $\mathrm{H} 4$ nearby facilitating DSB repair (Fig. 7).

In this study, we applied genetic interaction analysis on a large scale as a general approach for analyzing the complexities of histone (de)acetylation in yeast. New functions of the NuA4 and HDA complexes were identified, and a potential nonhistone substrate of Esalp and $\mathrm{Rpd} 3 \mathrm{p}$ was found. Extensions of this powerful strategy to mammalian systems will certainly be of interest in light of recent advances in high-throughput methodologies based on RNA interference (Silva et al. 2008).

\section{Materials and methods}

Full experimental details and data analysis methods are provided in the Supplemental Material. 


\section{Ts alleles creation}

The method to generate Ts alleles was performed as described (Huang et al. 2008). To construct a cloning vector, the $5^{\prime}$ promoter and $3^{\prime}$ terminator regions of an essential gene were cloned into a CEN plasmid with URA3 marker. A pool of mutations was generated in a cassette containing the promoter, the open reading frame, and the terminator regions by manganese-driven errorprone PCR reaction. The mutagenized PCR products and the digested cloning vector (to create a gap between the promoter and the terminator) were then cotransformed into a haploidconvertible heterozygous YKO strain of the corresponding essential gene. Vectors harboring mutated cassettes were generated by recombinational gap repair. Cells containing a library of mutations were sporulated and then subjected to selection for $\mathrm{Ura}^{+}-\mathrm{G} 418^{\mathrm{R}}$ phenotype in magic medium without uracil (MM-Ura; SC-LeuHis-Arg-Ura + canavanine + G418). These spores were then incubated at $25^{\circ} \mathrm{C}$ or $37^{\circ} \mathrm{C}$ to screen for a Ts phenotype.

\section{dSLAM and genetic interaction target gene validation}

The synthetic lethality screen was performed as described previously (Pan et al. 2006). A pool of haploid-convertible heterozygous diploid YKO library was transformed either once with a $U R A 3$ knockout cassette for nonessential query genes, or sequentially with a natMX knockout cassette followed by YCplac33 harboring a Ts allele for essential query genes. The resulting heterozygous double-mutant pool was then subjected to selection for a mixed population of single and double mutants as the control pool and a pure population of double mutants as the experimental pool in magic medium with the appropriate combination of selecting drugs. Three different semipermissive temperatures were tested for optimizing the screen conditions for essential query genes. Strains with a control/experiment ratio $\geq 2$ in either UPTAG or DNTAG were selected for validation either with random spore analysis or tetrad dissection.

\section{Data access}

Microarray data were submitted to GEO with accession code GSE9771.

\section{URLS}

BioGRID database: http://www.thebiogrid.org; MIPS database: http://mips.gsf.de; Saccharomyces genome database: http://www. yeastgenome.org.

\section{Acknowledgments}

We thank W. Zachariae for providing the $c d c 20-1$ Ts allele; E.M. Cooper and J. Dai for gifts of reagents; members of the Boeke laboratory for valuable discussions throughout the course of the work; and P.B. Meluh, A. Norris, K.A. O'Donnell, and O.J. Rando for their critical comments on the manuscript. Y.Q. is an IBM predoctoral fellow. X.P. was a fellow of the Leukemia and Lymphoma Society. This work was supported by NIH Roadmap grant "Technology Center for Networks and Pathways" (U54 RR 020839) and grant R01 HG 02432 to J.D.B.

\section{References}

Akhtar, A. and Gasser, S.M. 2007. The nuclear envelope and transcriptional control. Nat. Rev. Genet. 8: 507-517.

Allard, S., Utley, R.T., Savard, J., Clarke, A., Grant, P., Brandl,
C.J., Pillus, L., Workman, J.L., and Cote, J. 1999. NuA4, an essential transcription adaptor/histone $\mathrm{H} 4$ acetyltransferase complex containing Esalp and the ATM-related cofactor Tralp. EMBO J. 18: 5108-5119.

Auger, A., Galarneau, L., Altaf, M., Nourani, A., Doyon, Y., Utley, R.T., Cronier, D., Allard, S., and Cote, J. 2008. Eaf1 is the platform for NuA4 molecular assembly that evolutionarily links chromatin acetylation to ATP-dependent exchange of histone H2A variants. Mol. Cell. Biol. 28: 22572270.

Babiarz, J.E., Halley, J.E., and Rine, J. 2006. Telomeric heterochromatin boundaries require NuA4-dependent acetylation of histone variant H2A.Z in Saccharomyces cerevisiae. Genes \& Dev. 20: 700-710.

Bird, A.W., Yu, D.Y., Pray-Grant, M.G., Qiu, Q., Harmon, K.E., Megee, P.C., Grant, P.A., Smith, M.M., and Christman, M.F. 2002. Acetylation of histone $\mathrm{H} 4$ by Esal is required for DNA double-strand break repair. Nature 419: 411-415.

Boudreault, A.A., Cronier, D., Selleck, W., Lacoste, N., Utley, R.T., Allard, S., Savard, J., Lane, W.S., Tan, S., and Cote, J. 2003. Yeast enhancer of polycomb defines global Esa1-dependent acetylation of chromatin. Genes \& Dev. 17: 14151428.

Carmen, A.A., Rundlett, S.E., and Grunstein, M. 1996. HDA1 and HDA3 are components of a yeast histone deacetylase (HDA) complex. J. Biol. Chem. 271: 15837-15844.

Carmen, A.A., Griffin, P.R., Calaycay, J.R., Rundlett, S.E., Suka, Y., and Grunstein, M. 1999. Yeast HOS3 forms a novel trichostatin A-insensitive homodimer with intrinsic histone deacetylase activity. Proc. Natl. Acad. Sci. 96: 12356-12361.

Carrozza, M.J., Li, B., Florens, L., Suganuma, T., Swanson, S.K., Lee, K.K., Shia, W.J., Anderson, S., Yates, J., Washburn, M.P., et al. 2005. Histone $\mathrm{H} 3$ methylation by Set2 directs deacetylation of coding regions by $\operatorname{Rpd} 3 \mathrm{~S}$ to suppress spurious intragenic transcription. Cell 123: 581-592.

Choy, J.S. and Kron, S.J. 2002. NuA4 subunit Yng2 function in intra-S-phase DNA damage response. Mol. Cell. Biol. 22: 8215-8225.

Clarke, A.S., Lowell, J.E., Jacobson, S.J., and Pillus, L. 1999. Esalp is an essential histone acetyltransferase required for cell cycle progression. Mol. Cell. Biol. 19: 2515-2526.

Collins, S.R., Miller, K.M., Maas, N.L., Roguev, A., Fillingham, J., Chu, C.S., Schuldiner, M., Gebbia, M., Recht, J., Shales, M., et al. 2007. Functional dissection of protein complexes involved in yeast chromosome biology using a genetic interaction map. Nature 446: 806-810.

Davierwala, A.P., Haynes, J., Li, Z., Brost, R.L., Robinson, M.D., Yu, L., Mnaimneh, S., Ding, H., Zhu, H., Chen, Y., et al. 2005. The synthetic genetic interaction spectrum of essential genes. Nat. Genet. 37: 1147-1152.

Denu, J.M. 2005. The Sir 2 family of protein deacetylases. Curr. Opin. Chem. Biol. 9: 431-440.

Dhillon, N., Oki, M., Szyika, S.J., Aparicio, O.M., and Kamakaka, R.T. 2006. H2A.Z functions to regulate progression through the cell cycle. Mol. Cell. Biol. 26: 489-501.

Downs, J.A., Allard, S., Jobin-Robitaille, O., Javaheri, A., Auger, A., Bouchard, N., Kron, S.J., Jackson, S.P., and Cote, J. 2004. Binding of chromatin-modifying activities to phosphorylated histone H2A at DNA damage sites. Mol. Cell 16: 979-990.

Doyon, Y. and Cote, J. 2004. The highly conserved and multifunctional NuA4 HAT complex. Curr. Opin. Genet. Dev. 14: $147-154$

Durant, M. and Pugh, B.F. 2006. Genome-wide relationships between TAF1 and histone acetyltransferases in Saccharomyces cerevisiae. Mol. Cell. Biol. 26: 2791-2802.

Eisen, M.B., Spellman, P.T., Brown, P.O., and Botstein, D. 1998. 
Cluster analysis and display of genome-wide expression patterns. Proc. Natl. Acad. Sci. 95: 14863-14868.

Galarneau, L., Nourani, A., Boudreault, A.A., Zhang, Y., Heliot, L., Allard, S., Savard, J., Lane, W.S., Stillman, D.J., and Cote, J. 2000. Multiple links between the NuA4 histone acetyltransferase complex and epigenetic control of transcription. Mol. Cell 5: 927-937.

Glozak, M.A. and Seto, E. 2007. Histone deacetylases and cancer. Oncogene 26: 5420-5432.

Hartman, J.L.T., Garvik, B., and Hartwell, L. 2001. Principles for the buffering of genetic variation. Science 291: 1001-1004.

Howe, L., Auston, D., Grant, P., John, S., Cook, R.G., Workman, J.L., and Pillus, L. 2001. Histone H3 specific acetyltransferases are essential for cell cycle progression. Genes \& Dev. 15: 3144-3154.

Huang, Z., Sucgang, R.S., Lin, Y.Y., Shi, X., Boeke, J.D., and Pan, X. 2008. Plasmid-chromosome shuffling for non-deletion alleles in yeast. Nat. Methods 5: 167-169.

Huisinga, K.L. and Pugh, B.F. 2004. A genome-wide housekeeping role for TFIID and a highly regulated stress-related role for SAGA in Saccharomyces cerevisiae. Mol. Cell 13: 573 585.

Keogh, M.C., Kurdistani, S.K., Morris, S.A., Ahn, S.H., Podolny, V., Collins, S.R., Schuldiner, M., Chin, K., Punna, T., Thompson, N.J., et al. 2005. Cotranscriptional set2 methylation of histone $\mathrm{H} 3$ lysine 36 recruits a repressive $\mathrm{Rpd} 3 \mathrm{com}$ plex. Cell 123: 593-605.

Keogh, M.C., Mennella, T.A., Sawa, C., Berthelet, S., Krogan, N.J., Wolek, A., Podolny, V., Carpenter, L.R., Greenblatt, J.F., Baetz, K., et al. 2006. The Saccharomyces cerevisiae histone H2A variant Htz1 is acetylated by NuA4. Genes \& Dev. 20: 660-665.

Kobor, M.S., Venkatasubrahmanyam, S., Meneghini, M.D., Gin, J.W., Jennings, J.L., Link, A.J., Madhani, H.D., and Rine, J. 2004. A protein complex containing the conserved Swi2/ Snf2-related ATPase Swrlp deposits histone variant H2A.Z into euchromatin. PLoS Biol. 2: E131. doi: 10.1371/journal. pbio.0020131.

Kouzarides, T. 2007. Chromatin modifications and their function. Cell 128: 693-705.

Krogan, N.J., Keogh, M.C., Datta, N., Sawa, C., Ryan, O.W., Ding, H., Haw, R.A., Pootoolal, J., Tong, A., Canadien, V., et al. 2003. A Snf2 family ATPase complex required for recruitment of the histone H2A variant Htz1. Mol. Cell 12: 15651576.

Krogan, N.J., Baetz, K., Keogh, M.C., Datta, N., Sawa, C., Kwok, T.C., Thompson, N.J., Davey, M.G., Pootoolal, J., Hughes, T.R., et al. 2004a. Regulation of chromosome stability by the histone H2A variant Htzl, the Swrl chromatin remodeling complex, and the histone acetyltransferase NuA4. Proc. Nat1. Acad. Sci. 101: 13513-13518.

Krogan, N.J., Lam, M.H., Fillingham, J., Keogh, M.C., Gebbia, M., Li, J., Datta, N., Cagney, G., Buratowski, S., Emili, A., et al. 2004b. Proteasome involvement in the repair of DNA double-strand breaks. Mol. Cell 16: 1027-1034.

Le Masson, I., Yu, D.Y., Jensen, K., Chevalier, A., Courbeyrette, R., Boulard, Y., Smith, M.M., and Mann, C. 2003. Yaf9, a novel NuA4 histone acetyltransferase subunit, is required for the cellular response to spindle stress in yeast. Mol. Cell. Biol. 23: 6086-6102.

Lee, K.K. and Workman, J.L. 2007. Histone acetyltransferase complexes: One size doesn't fit all. Nat. Rev. Mol. Cell Biol. 8: $284-295$

Lee, S.E., Moore, J.K., Holmes, A., Umezu, K., Kolodner, R.D., and Haber, J.E. 1998. Saccharomyces Ku70, mre11/rad50 and RPA proteins regulate adaptation to G2/M arrest after DNA damage. Cell 94: 399-409.

Li, B., Gogol, M., Carey, M., Lee, D., Seidel, C., and Workman, J.L. 2007. Combined action of PHD and chromo domains directs the Rpd3S HDAC to transcribed chromatin. Science 316: 1050-1054

Meneghini, M.D., Wu, M., and Madhani, H.D. 2003. Conserved histone variant H2A.Z protects euchromatin from the ectopic spread of silent heterochromatin. Cell 112: 725-736.

Millar, C.B. and Grunstein, M. 2006. Genome-wide patterns of histone modifications in yeast. Nat. Rev. Mol. Cell Biol. 7: 657-666.

Millar, C.B., Xu, F., Zhang, K., and Grunstein, M. 2006. Acetylation of $\mathrm{H} 2 \mathrm{AZ}$ Lys 14 is associated with genome-wide gene activity in yeast. Genes \& Dev. 20: 711-722.

Mitchell, L., Lambert, J.P., Gerdes, M., Al-Madhoun, A.S., Skerjanc, I.S., Figeys, D., and Baetz, K. 2008. Functional dissection of the NuA4 histone acetyltransferase reveals its role as a genetic hub and that Eaf1 is essential for complex integrity. Mol. Cell. Biol. 28: 2244-2256.

Mizuguchi, G., Shen, X., Landry, J., Wu, W.H., Sen, S., and Wu, C. 2004. ATP-driven exchange of histone H2AZ variant catalyzed by SWR1 chromatin remodeling complex. Science 303: 343-348.

Pan, X., Ye, P., Yuan, D.S., Wang, X., Bader, J.S., and Boeke, J.D. 2006. A DNA integrity network in the yeast Saccharomyces cerevisiae. Cell 124: 1069-1081.

Parsons, A.B., Brost, R.L., Ding, H., Li, Z., Zhang, C., Sheikh, B., Brown, G.W., Kane, P.M., Hughes, T.R., and Boone, C. 2004. Integration of chemical-genetic and genetic interaction data links bioactive compounds to cellular target pathways. Nat. Biotechnol. 22: 62-69.

Rahl, P.B., Chen, C.Z., and Collins, R.N. 2005. Elplp, the yeast homolog of the FD disease syndrome protein, negatively regulates exocytosis independently of transcriptional elongation. Mol. Cell 17: 841-853.

Robyr, D., Suka, Y., Xenarios, I., Kurdistani, S.K., Wang, A., Suka, N., and Grunstein, M. 2002. Microarray deacetylation maps determine genome-wide functions for yeast histone deacetylases. Cell 109: 437-446.

Santisteban, M.S., Kalashnikova, T., and Smith, M.M. 2000. Histone H2A.Z regulats transcription and is partially redundant with nucleosome remodeling complexes. Cell 103: 411-422.

Schuldiner, M., Collins, S.R., Thompson, N.J., Denic, V., Bhamidipati, A., Punna, T., Ihmels, J., Andrews, B., Boone, C., Greenblatt, J.F., et al. 2005. Exploration of the function and organization of the yeast early secretory pathway through an epistatic miniarray profile. Cell 123: 507-519.

Segre, D., Deluna, A., Church, G.M., and Kishony, R. 2005. Modular epistasis in yeast metabolism. Nat. Genet. 37: 7783.

Selleck, W., Fortin, I., Sermwittayawong, D., Cote, J., and Tan, S. 2005. The Saccharomyces cerevisiae Piccolo NuA4 histone acetyltransferase complex requires the Enhancer of Polycomb A domain and chromodomain to acetylate nucleosomes. Mol. Cell. Biol. 25: 5535-5542.

Shahbazian, M.D. and Grunstein, M. 2007. Functions of sitespecific histone acetylation and deacetylation. Annu. Rev. Biochem. 76: 75-100.

Silva, J.M., Marran, K., Parker, J.S., Silva, J., Golding, M., Schlabach, M.R., Elledge, S.J., Hannon, G.J., and Chang, K. 2008. Profiling essential genes in human mammary cells by multiplex RNAi screening. Science 319: 617-620.

Smith, E.R., Eisen, A., Gu, W., Sattah, M., Pannuti, A., Zhou, J., Cook, R.G., Lucchesi, J.C., and Allis, C.D. 1998. ESA1 is a histone acetyltransferase that is essential for growth in 
Lin et al.

yeast. Proc. Nat1. Acad. Sci. 95: 3561-3565.

Takahashi, H., McCaffery, J.M., Irizarry, R.A., and Boeke, J.D. 2006. Nucleocytosolic acetyl-coenzyme a synthetase is required for histone acetylation and global transcription. Mol. Cell 23: 207-217.

Tamburini, B.A. and Tyler, J.K. 2005. Localized histone acetylation and deacetylation triggered by the homologous recombination pathway of double-strand DNA repair. Mol. Cell. Biol. 25: 4903-4913.

Tong, A.H., Lesage, G., Bader, G.D., Ding, H., Xu, H., Xin, X., Young, J., Berriz, G.F., Brost, R.L., Chang, M., et al. 2004. Global mapping of the yeast genetic interaction network. Science 303: 808-813.

Tsukuda, T., Fleming, A.B., Nickoloff, J.A., and Osley, M.A. 2005. Chromatin remodelling at a DNA double-strand break site in Saccharomyces cerevisiae. Nature 438: 379-383.

Verma, R., Aravind, L., Oania, R., McDonald, W.H., Yates 3rd, J.R., Koonin, E.V., and Deshaies, R.J. 2002. Role of Rpn11 metalloprotease in deubiquitination and degradation by the 26S proteasome. Science 298: 611-615.

Vogelauer, M., Wu, J., Suka, N., and Grunstein, M. 2000. Global histone acetylation and deacetylation in yeast. Nature 408: 495-498.

Wu, J., Suka, N., Carlson, M., and Grunstein, M. 2001. TUP1 utilizes histone H3/H2B-specific HDA1 deacetylase to repress gene activity in yeast. Mol. Cell 7: 117-126.

$\mathrm{Xu}$, W.S., Parmigiani, R.B., and Marks, P.A. 2007. Histone deacetylase inhibitors: Molecular mechanisms of action. Oncogene 26: 5541-5552.

Zhang, W., Bone, J.R., Edmondson, D.G., Turner, B.M., and Roth, S.Y. 1998. Essential and redundant functions of histone acetylation revealed by mutation of target lysines and loss of the Gcn5p acetyltransferase. EMBO J. 17: 3155-3167.

Zhang, H., Richardson, D.O., Roberts, D.N., Utley, R., Erdjument-Bromage, H., Tempst, P., Cote, J., and Cairns, B.R. 2004. The Yaf9 component of the SWR 1 and NuA4 complexes is required for proper gene expression, histone $\mathrm{H} 4$ acetylation, and Htzl replacement near telomeres. Mol. Cell. Biol. 24: 9424-9436. 


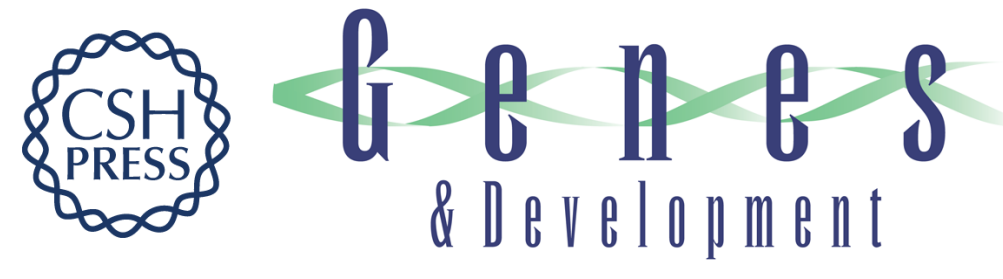

\section{A comprehensive synthetic genetic interaction network governing yeast histone acetylation and deacetylation}

Yu-yi Lin, Yan Qi, Jin-ying Lu, et al.

Genes Dev. 2008, 22:

Access the most recent version at doi:10.1101/gad.1679508

Supplemental http://genesdev.cshlp.org/content/suppl/2008/08/05/22.15.2062.DC1
Material

References This article cites 62 articles, 28 of which can be accessed free at:

http://genesdev.cshlp.org/content/22/15/2062.full.html\#ref-list-1

License

Email Alerting

Receive free email alerts when new articles cite this article - sign up in the box at the top

Service

right corner of the article or click here.

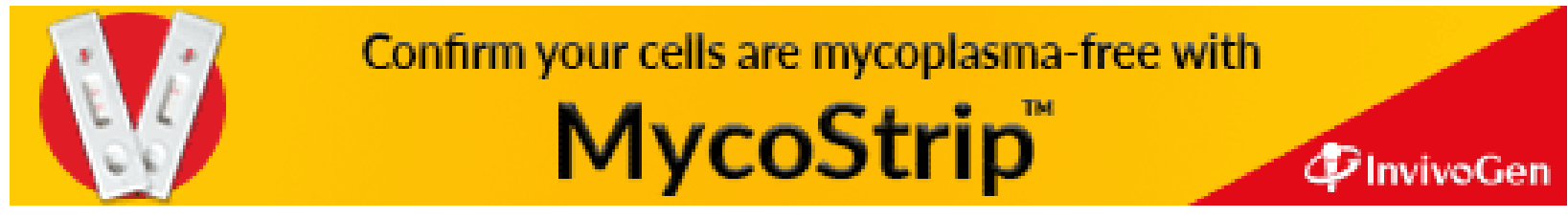

